UCSD/PTH 98-01

DESY 98-008

hep-ph/9801397

January 1998

\title{
Top Quark Pair Production at Threshold: Complete Next-to-next-to-leading Order Relativistic Corrections
}

\author{
A. H. Hoang ${ }^{a}$ and T. Teubner ${ }^{b}$ \\ a Department of Physics, University of California, San Diego, \\ La Jolla, CA 92093-0319, USA \\ ${ }^{b}$ Deutsches Elektronen-Synchrotron DESY, \\ D-22603 Hamburg, Germany
}

\begin{abstract}
The complete next-to-next-to-leading order (i.e. $\mathcal{O}\left(v^{2}\right), \mathcal{O}\left(v \alpha_{s}\right)$ and $\left.\mathcal{O}\left(\alpha_{s}^{2}\right)\right)$ relativistic corrections to the total photon mediated $t \bar{t}$ production cross section at threshold are presented in the framework of nonrelativistic quantum chromodynamics. The results are obtained using semi-analytic methods and "direct matching". The size of the next-to-next-to-leading order relativistic corrections is found to be comparable to the size of the next-to-leading order ones.
\end{abstract}

PACS numbers: 14.65.Ha, 13.85.Lg, 12.38.Bx. 


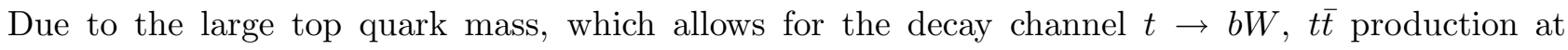
threshold in lepton pair collisions offers the unique opportunity to study heavy quark-antiquark bound state and near threshold dynamics using perturbative QCD [1]. With this motivation in mind a considerable number of studies has been carried out in the past in order to calculate $t \bar{t}$ production observables [2, 3, 4, 5, 6] and explore their potential for measurements of the top quark mass $M_{t}$ and the strong coupling $\alpha_{s}$ at future experiments like the LC (Linear Collider) [7] or the FMC (First Muon Collider) [8]. In view of the high precision which might be achieved for the QCD calculations as well as for $t \bar{t}$ production measurements even relatively small effects coming from a light Higgs boson [2, 9] have been investigated. However, the present day analyses only include QCD effects up to next-to-leading order (NLO) in form of the one-loop corrections to the QCD potential 110, 11] and various $\mathcal{O}\left(\alpha_{s}\right)$ shortdistance corrections. A complete next-to-next-to-leading order (NNLO) calculation, which would be necessary to study the reliability of the present day analyses and to make the consideration of small effects from beyond QCD at all feasible, has been missing so far.

In this letter we present the complete NNLO relativistic corrections to the total photon mediated $t \bar{t}$ production cross section. As NNLO we count all corrections of order $v^{2}, v \alpha_{s}$ and $\alpha_{s}^{2}$ relative to the cross section in the nonrelativistic limit, $v$ being the c.m. velocity of the top quarks. As relativistic, on the other hand, we count the corrections coming from the top quark kinetic energy, the $t \bar{t}$ production and annihilation process including the short-distance corrections, and the $t \bar{t}$ interaction potentials. We use NRQCD [12] to conveniently parameterize calculations and results in a systematic manner following the approach proposed in [13]. The calculations are carried out using semi-analytic methods and the "direct matching" procedure introduced in [14]. We would like to point out that NNLO corrections involving the top quark decay are not determined here. The latter effects would include $\mathcal{O}\left(\alpha_{s}^{2}\right)$ two loop corrections to the free top quark width and a consistent treatment of the effects from the off-shellness of the decaying top quarks, the time dilatation and the interactions among the decay products and the other top quark (if it is not decayed yet). Although the size and the interplay of all these effects have been studied at various places in the literature (see e.g. [5, 15, 16]), their consistent treatment at NNLO still remains an open problem. As far as the NNLO relativistic corrections discussed in this letter are concerned we will use the naive replacement

$$
E \equiv \sqrt{s}-2 M_{t} \longrightarrow \tilde{E}=E+i \Gamma_{t}
$$

in the spirit of [1] in order to examine their size and properties, where $\Gamma_{t}$ represents a constant which is not necessarily the decay width of a free top quark. We also would like to emphasize that we treat all interactions purely perturbatively and that nowhere in this work the confining long-range contributions to the QCD potential or other nonperturbative effects are taken into account. This is somewhat contrary to the standard present day approach used to describe $t \bar{t}$ production at threshold (see [2, 3, 4, 5]), but we take the position that nonperturbative effects might be added later as a correction.

We start from the NRQCD Lagrangian

$$
\begin{aligned}
& \mathcal{L}_{\mathrm{NRQCD}}=-\frac{1}{2} \operatorname{Tr} G^{\mu \nu} G_{\mu \nu}+\sum_{q=u, d, s, c, b} \bar{q} i \not D q+\psi^{\dagger}\left[i D_{t}+a_{1} \frac{\boldsymbol{D}^{2}}{2 M_{t}}+a_{2} \frac{\boldsymbol{D}^{4}}{8 M_{t}^{3}}\right] \psi+\ldots \\
&+\psi^{\dagger}\left[\frac{a_{3} g_{s}}{2 M_{t}} \boldsymbol{\sigma} \cdot \boldsymbol{B}+\frac{a_{4} g_{s}}{8 M_{t}^{2}}(\boldsymbol{D} \cdot \boldsymbol{E}-\boldsymbol{E} \cdot \boldsymbol{D})+\frac{a_{5} g_{s}}{8 M_{t}^{2}} i \boldsymbol{\sigma}(\boldsymbol{D} \times \boldsymbol{E}-\boldsymbol{E} \times \boldsymbol{D})\right] \psi+\ldots
\end{aligned}
$$

\footnotetext{
${ }^{1}$ Throughout this paper $M_{t}$ is understood as the top quark pole mass.
} 
The gluonic and light quark degrees of freedom are described by the conventional relativistic Lagrangian, whereas the top and antitop quark are described by the Pauli spinors $\psi$ and $\chi$, respectively. For convenience all color indices are suppressed. The straightforward antitop bilinears are omitted and only those terms relevant for the NNLO cross section are displayed. $D_{t}$ and $\boldsymbol{D}$ are the time and space components of the gauge covariant derivative $D_{\mu}$, and $E^{i}=G^{0 i}$ and $B^{i}=\frac{1}{2} \epsilon^{i j k} G^{j k}$ the electric and magnetic components of the gluon field strength tensor (in Coulomb gauge). The short-distance coefficients $a_{1}, \ldots, a_{5}$ are normalized to one at the Born level. Because we use "direct matching" 14, their higher order contributions are irrelevant for this work.

To formulate the normalized total $t \bar{t}$ production cross section (via a virtual photon) $R=$ $\sigma\left(\begin{array}{c}e^{+} e^{-} \\ \mu^{+} \mu^{-}\end{array} \rightarrow \gamma^{*} \rightarrow t \bar{t}\right) / \sigma_{p t}\left(\sigma_{p t}=4 \pi \alpha^{2} / 3 s\right)$ in the nonrelativistic region at NNLO in NRQCD we start from the fully covariant expression for the cross section

$$
R\left(q^{2}\right)=\frac{4 \pi Q_{t}^{2}}{q^{2}} \operatorname{Im}\left[\left\langle 0\left|T \tilde{j}_{\mu}(q) \tilde{j}^{\mu}(-q)\right| 0\right\rangle\right],
$$

where $Q_{t}=2 / 3$ is the top quark electric charge. We then expand the electromagnetic current (in momentum space) $\tilde{j}_{\mu}( \pm q)=\left(\tilde{\bar{t}} \gamma^{\mu} \tilde{t}\right)( \pm q)$ which produces/annihilates a $t \bar{t}$ pair with c.m. energy $\sqrt{q^{2}}$ in terms of ${ }^{3} S_{1}$ NRQCD currents up to dimension eight $(i=1,2,3)$

$$
\tilde{j}_{i}(q)=b_{1}\left(\tilde{\psi}^{\dagger} \sigma_{i} \tilde{\chi}\right)(q)-\frac{b_{2}}{6 M_{t}^{2}}\left(\tilde{\psi}^{\dagger} \sigma_{i}\left(-\frac{i}{2} \stackrel{\leftrightarrow}{\boldsymbol{D}}\right) \tilde{\chi}\right)(q)+\ldots
$$

where the constants $b_{1}$ and $b_{2}$ are short-distance coefficients normalized to one at the Born level. The expansion of $\tilde{j}_{i}(-q)$ is obtained from Eq. (4) via charge conjugation symmetry. It should be noted that only the spatial components of the currents contribute. Inserting expansion (目) back into Eq. (3) leads to the NRQCD expression of the nonrelativistic cross section at the NNLO level

$$
\begin{aligned}
R_{\mathrm{NNLO}}^{\mathrm{thr}}(\tilde{E})= & \frac{\pi Q_{t}^{2}}{M_{t}^{2}} C_{1}\left(\mu_{\mathrm{hard}}, \mu_{\mathrm{fac}}\right) \operatorname{Im}\left[\mathcal{A}_{1}\left(\tilde{E}, \mu_{\mathrm{soft}}, \mu_{\mathrm{fac}}\right)\right] \\
& -\frac{4 \pi Q_{t}^{2}}{3 M_{t}^{4}} C_{2}\left(\mu_{\mathrm{hard}}, \mu_{\mathrm{fac}}\right) \operatorname{Im}\left[\mathcal{A}_{2}\left(\tilde{E}, \mu_{\mathrm{soft}}, \mu_{\mathrm{fac}}\right)\right]+\ldots,
\end{aligned}
$$

where

$$
\begin{aligned}
& \mathcal{A}_{1}=\left\langle 0\left|\left(\tilde{\psi}^{\dagger} \vec{\sigma} \tilde{\chi}\right)\left(\tilde{\chi}^{\dagger} \vec{\sigma} \tilde{\psi}\right)\right| 0\right\rangle \\
& \mathcal{A}_{2}=\frac{1}{2}\langle 0|\left(\tilde{\psi}^{\dagger} \vec{\sigma} \tilde{\chi}\right)\left(\tilde{\chi}^{\dagger} \vec{\sigma}\left(-\frac{i}{2} \stackrel{\leftrightarrow}{\boldsymbol{D}}\right) \tilde{\psi}\right)+\text { h.c. }|0\rangle
\end{aligned}
$$

To obtain the factor $4 \pi Q_{t}^{2} / 3 M_{t}^{4}$ in the second line of expression (5) we have already used relation (14). The cross section is expanded in terms of a sum of absorptive parts of nonrelativistic current correlators (containing long-distance physics [) multiplied by short-distance coefficients $C_{i}(i=1,2, \ldots)$. In Eq. (5) we have also shown the dependences on the various renormalization scales: the soft scale $\mu_{\text {soft }}$ and the hard scale $\mu_{\text {hard }}$ are governing the perturbative expansions of the correlators and the short-

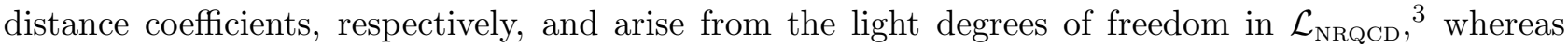
the factorization scale $\mu_{\text {fac }}$ essentially represents the boundary between hard (i.e. of order $M_{t}$ ) and

\footnotetext{
${ }^{2}$ In the context of this paper "long-distance" is not equivalent to "nonperturbative".

3 Throughout this work we use the convention $\alpha_{s}=\alpha_{s}^{\left(n_{l}=5\right)}$ in the $\overline{\mathrm{MS}}$ scheme.
} 
soft momenta. This boundary is not uniquely defined and therefore both, the correlators and the short-distance coefficients, in general depend on $\mu_{\mathrm{fac}}$ (leading to new anomalous dimensions). Because the term in the second line in Eq. (5) is already of NNLO (i.e. suppressed by $v^{2}$ ) we can set $C_{2}=1$ and ignore the factorization scale dependence of the correlator $\mathcal{A}_{2}$. The calculation of all terms in expression (5) proceeds in two basic steps.

Step 1: Calculation of the nonrelativistic correlators. - The calculation of the correlators at NNLO is simplified enormously by the fact that for the $t \bar{t}$ pair, which is produced and annihilated in a color singlet S-wave state, all retardation effects can be neglected. Technically this means that the transverse gluon exchange, which would lead to temporally retarded interactions (closely related to Lamb-shift type corrections known in QED), can be treated as instantaneous, i.e. its energy dependence can be ignored. This can be seen by using formal counting rules (see e.g. [17, 18]) or from physical considerations because a real gluon (i.e. one that carries energy) which is radiated after the $t \bar{t}$ pair was produced has to be absorbed before the $t \bar{t}$ pair is annihilated. Phase space effects and the dipole matrix element then lead to a suppression of this process $\propto v^{3}$, i.e. to an effect beyond NNLO. In other words, as far as the nonrelativistic correlators in Eq. (5) are concerned, NRQCD reduces to a two-body (top-antitop) Schrödinger theory. The potentials in the resulting Schrödinger equation are determined by considering $t \bar{t} \rightarrow t \bar{t}$ one gluon exchange t-channel scattering amplitudes in NRQCD. To NNLO (i.e. including potentials suppressed by at most $\alpha_{s}^{2}, \alpha_{s} / M_{t}$ or $1 / M_{t}^{2}$ relative to the Coulomb potential) the relevant potentials $\operatorname{read}\left(a_{s} \equiv \alpha_{s}\left(\mu_{\text {soft }}\right), C_{A}=3, C_{F}=4 / 3, T=1 / 2, \tilde{\mu} \equiv e^{\gamma} \mu_{\text {soft }}\right.$, $r \equiv|\vec{r}|)$

$$
\begin{aligned}
V_{c}(\vec{r})= & -\frac{C_{F} a_{s}}{r}\left\{1+\left(\frac{a_{s}}{4 \pi}\right)\left[2 \beta_{0} \ln (\tilde{\mu} r)+a_{1}\right]\right. \\
& \left.+\left(\frac{a_{s}}{4 \pi}\right)^{2}\left[\beta_{0}^{2}\left(4 \ln ^{2}(\tilde{\mu} r)+\frac{\pi^{2}}{3}\right)+2\left(2 \beta_{0} a_{1}+\beta_{1}\right) \ln (\tilde{\mu} r)+a_{2}\right]\right\}, \\
V_{\mathrm{BF}}(\vec{r})= & \frac{C_{F} a_{s} \pi}{M_{t}^{2}}\left[1+\frac{8}{3} \vec{S}_{t} \vec{S}_{\bar{t}}\right] \delta^{(3)}(\vec{r})+\frac{C_{F} a_{s}}{2 M_{t}^{2} r}\left[\vec{\nabla}^{2}+\frac{1}{r^{2}} \vec{r}(\vec{r} \vec{\nabla}) \vec{\nabla}\right] \\
& -\frac{3 C_{F} a_{s}}{M_{t}^{2} r^{3}}\left[\frac{1}{3} \vec{S}_{t} \vec{S}_{\bar{t}}-\frac{1}{r^{2}}\left(\vec{S}_{t} \vec{r}\right)\left(\vec{S}_{\bar{t}} \vec{r}\right)\right]+\frac{3 C_{F} a_{s}}{2 M_{t}^{2} r^{3}} \vec{L}\left(\vec{S}_{t}+\vec{S}_{\bar{t}}\right), \\
V_{\mathrm{NA}}(\vec{r})= & -\frac{C_{A} C_{F} a_{s}^{2}}{2 M_{t} r^{2}},
\end{aligned}
$$

where $\vec{S}_{t}$ and $\vec{S}_{\bar{t}}$ are the top and antitop spin operators and $\vec{L}$ is the angular momentum operator and $\left(n_{l}=5\right)$

$$
\begin{aligned}
& \beta_{0}=\frac{11}{3} C_{A}-\frac{4}{3} T n_{l}, \\
& \beta_{1}=\frac{34}{3} C_{A}^{2}-\frac{20}{3} C_{A} T n_{l}-4 C_{F} T n_{l}, \\
& a_{1}=\frac{31}{9} C_{A}-\frac{20}{9} T n_{l}, \\
& a_{2}=\left(\frac{4343}{162}+6 \pi^{2}-\frac{\pi^{4}}{4}+\frac{22}{3} \zeta_{3}\right) C_{A}^{2}-\left(\frac{1798}{81}+\frac{56}{3} \zeta_{3}\right) C_{A} T n_{l}
\end{aligned}
$$




$$
-\left(\frac{55}{3}-16 \zeta_{3}\right) C_{F} T n_{l}+\left(\frac{20}{9} T n_{l}\right)^{2} .
$$

The constants $\beta_{0}$ and $\beta_{1}$ are the one- and two-loop coefficients of the QCD beta function and $\gamma=0.577216 \ldots$ is the Euler constant. $V_{c}$ is the Coulomb (static) potential. Its $\mathcal{O}\left(\alpha_{s}\right)$ and $\mathcal{O}\left(\alpha_{s}^{2}\right)$ corrections have been determined in [10, 11] and [19], respectively. $V_{\mathrm{BF}}$ is the Breit-Fermi potential known from positronium and $V_{\mathrm{NA}}$ a purely non-Abelian potential generated through non-analytic terms in one-loop NRQCD (or QCD) diagrams containing the triple gluon vertex (see e.g. [20] for older references). The nonrelativistic correlators are directly related to the Green function of the Schrödinger equation

$$
\left(-\frac{\vec{\nabla}^{2}}{M_{t}}-\frac{\vec{\nabla}^{4}}{4 M_{t}^{3}}+V_{c}(\vec{r})+V_{\mathrm{BF}}(\vec{r})+V_{\mathrm{NA}}(\vec{r})-\tilde{E}\right) G\left(\vec{r}, \vec{r}^{\prime}, \tilde{E}\right)=\delta^{(3)}\left(\vec{r}-\vec{r}^{\prime}\right),
$$

where $V_{\mathrm{BF}}$ is evaluated for the ${ }^{3} S_{1}$ configuration only. The correlators read

$$
\begin{aligned}
& \mathcal{A}_{1}=6 N_{c}\left[\lim _{|\vec{r}|,\left|\vec{r}^{\prime}\right| \rightarrow 0} G\left(\vec{r}, \vec{r}^{\prime}, \tilde{E}\right)\right], \\
& \mathcal{A}_{2}=M_{t} \tilde{E} \mathcal{A}_{1} .
\end{aligned}
$$

Relation (13) can be easily inferred by taking into account that the Green function $G(\vec{r}, \vec{r}, \tilde{E})$ describes the propagation of a top-antitop pair which is produced and annihilated at distances $|\vec{r}|$ and $|\vec{r}|$, respectively. Because the exact solution of Eq. (12) seems to be an impossible task, we rely on a numerical solution of the equation

$$
\left(-\frac{\vec{\nabla}^{2}}{M_{t}}+V_{c}(\vec{r})-\tilde{E}\right) G_{c}\left(\vec{r}, \vec{r}^{\prime}, \tilde{E}\right)=\delta^{(3)}\left(\vec{r}-\vec{r}^{\prime}\right)
$$

using techniques developed in [2, 4]. The result for $G_{c}(0,0, \tilde{E})$ is then combined with the corrections to the leading order (LO) Coulomb Green function $G_{c}^{(0)}$ [21] (defined through Eq. (15) for $V_{c}(\vec{r})=$ $\left.-C_{F} a_{s} / r\right)$ coming from the kinetic energy correction and the potentials $V_{\mathrm{BF}}$ and $V_{\mathrm{NA}}$. These corrections are calculated analytically using Rayleigh-Schrödinger time-independent perturbation theory,

$$
\delta G\left(\vec{r}, \vec{r}^{\prime}, \tilde{E}\right)=\int d \vec{x}^{3} G_{c}^{(0)}(\vec{r}, \vec{x}, \tilde{E})\left[\frac{\vec{\nabla}^{4}}{4 M_{t}^{3}}-V_{\mathrm{BF}}(\vec{x})-V_{\mathrm{NA}}(\vec{x})\right] G_{c}^{(0)}\left(\vec{x}, \vec{r}^{\prime}, \tilde{E}\right) .
$$

For the calculation of Eq. (16) we use techniques employed in [22], where the Abelian NNLO contributions have already been determined. The final result for $\mathcal{A}_{1}$ at NNLO reads

$$
\begin{aligned}
\mathcal{A}_{1} & =6 N_{c}\left[G_{c}(0,0, \tilde{E})-G_{c}^{(0)}(0,0, \tilde{E})\right] \\
& +\frac{N_{c} C_{F} a_{s} M_{t}^{2}}{2 \pi}\left(1+\frac{3}{2} \frac{C_{A}}{C_{F}}\right)\left\{i \tilde{v}-C_{F} a_{s}\left[\ln \left(-i \frac{M_{t} \tilde{v}}{\mu_{\mathrm{fac}}}\right)+\gamma+\Psi\left(1-i \frac{C_{F} a_{s}}{2 \tilde{v}}\right)\right]\right\}^{2} \\
& +\frac{3 N_{c} M_{t}^{2}}{2 \pi}\left\{i \tilde{v}\left(1+\frac{5}{8} \tilde{v}^{2}\right)-C_{F} a_{s}\left(1+2 \tilde{v}^{2}\right)\left[\ln \left(-i \frac{M_{t} \tilde{v}}{\mu_{\mathrm{fac}}}\right)+\gamma+\Psi\left(1-i \frac{C_{F} a_{s}\left(1+\frac{11}{8} \tilde{v}^{2}\right)}{2 \tilde{v}}\right)\right]\right\},
\end{aligned}
$$

where

$$
\tilde{v} \equiv \sqrt{\frac{\tilde{E}}{M_{t}}}
$$


and $\Psi$ is the digamma function, $\Psi(z) \equiv \frac{d}{d z} \ln \Gamma(z)$. In the first line of Eq. (17) the LO Green function has been subtracted to avoid double counting of the LO contribution contained in the third line. It should be noted that the limit $|\vec{r}|,\left|\vec{r}^{\prime}\right| \rightarrow 0$ in expression (16) causes UV divergences which are regulated using the short-distance cutoff $\mu_{\mathrm{fac}}$. Further, all power divergences $\propto \mu_{\mathrm{fac}} / M_{t}$ are subtracted [23] and $\mu_{\text {fac }}$ is defined in a way that expression (17) takes the simple form shown above. The corresponding result in any other regularization scheme could be obtained from the one presented here through a redefinition of the factorization scale. For $\mathcal{A}_{2}$ only the LO contribution in Eq. (17) is relevant and we arrive at

$$
\mathcal{A}_{2}=\tilde{v}^{2} \frac{3 N_{c} M_{t}^{4}}{2 \pi}\left\{i \tilde{v}-C_{F} a_{s}\left[\ln \left(-i \frac{M_{t} \tilde{v}}{\mu_{\mathrm{fac}}}\right)+\gamma+\Psi\left(1-i \frac{C_{F} a_{s}}{2 \tilde{v}}\right)\right]\right\}
$$

There are no non-Abelian contributions to $\mathcal{A}_{2}$.

Step 2: Matching calculation. - The contributions to $C_{1}$ up to $\mathcal{O}\left(\alpha_{s}^{2}\right)$ are determined by matching expression (5) directly to the two-loop cross section calculated in full QCD in the (formal) limit $\alpha_{s} \ll v \ll 1$ for stable quarks $\left(\Gamma_{t}=0\right)$ keeping terms up to $\alpha_{s}^{2}$ and NNLO in the expansion in $v$ and setting $\mu_{\text {soft }}=\mu_{\text {hard }}$. To obtain the contributions $\propto \alpha_{s}^{2}$ originating from the potential $V_{c}$ in this limit we also employ time-independent perturbation theory in analogy to Eq. (16). The corresponding cross section in full QCD reads $\left(a_{h} \equiv \alpha_{s}\left(\mu_{\text {hard }}\right), v \equiv\left(E / M_{t}\right)^{1 / 2}\right)$

$$
\begin{aligned}
& R_{2 \text { loop } Q \mathrm{ND}}^{\mathrm{NNLO}}=N_{c} Q_{t}^{2}\left\{\left[\frac{3}{2} v-\frac{17}{16} v^{3}\right]+\frac{C_{F} a_{h}}{\pi}\left[\frac{3 \pi^{2}}{4}-6 v+\frac{\pi^{2}}{2} v^{2}\right]\right. \\
&+a_{h}^{2} {\left[\frac{C_{F}^{2} \pi^{2}}{8 v}+\frac{3}{2} C_{F}\left(-2 C_{F}+C_{A}\left(-\frac{11}{24} \ln \frac{4 v^{2} M_{t}^{2}}{\mu_{\text {hard }}^{2}}+\frac{31}{72}\right)+T n_{l}\left(\frac{1}{6} \ln \frac{4 v^{2} M_{t}^{2}}{\mu_{\text {hard }}^{2}}-\frac{5}{18}\right)\right)\right.} \\
&\left.\left.+\left(\frac{49 C_{F}^{2} \pi^{2}}{192}+\frac{3}{2} \kappa+\frac{C_{F}}{\pi^{2}}\left(\frac{11}{2} C_{A}-2 T n_{l}\right) \ln \frac{M_{t}^{2}}{\mu_{\text {hard }}^{2}}-C_{F}\left(C_{F}+\frac{3}{2} C_{A}\right) \ln v\right) v\right]\right\},
\end{aligned}
$$

where

$$
\begin{aligned}
\kappa= & C_{F}^{2}\left[\frac{1}{\pi^{2}}\left(\frac{39}{4}-\zeta_{3}\right)+\frac{4}{3} \ln 2-\frac{35}{18}\right]-C_{A} C_{F}\left[\frac{1}{\pi^{2}}\left(\frac{151}{36}+\frac{13}{2} \zeta_{3}\right)+\frac{8}{3} \ln 2-\frac{179}{72}\right] \\
& +C_{F} T\left[\frac{4}{9}\left(\frac{11}{\pi^{2}}-1\right)\right]+C_{F} T n_{l}\left[\frac{11}{9 \pi^{2}}\right] .
\end{aligned}
$$

The Born and $\mathcal{O}\left(\alpha_{s}\right)$ [24] contributions are standard. At order $\alpha_{s}^{2}$ the contributions in Eq. (20) proportional to $C_{F}^{2}, C_{A} C_{F}, C_{F} T n_{l}$ and $C_{F} T$ have been calculated in [25], 26], 27, 28] and [27, 29], respectively. The result for $C_{1}$ reads

$$
C_{1}=1-4 C_{F} \frac{a_{h}}{\pi}+a_{h}^{2}\left[\kappa+\frac{C_{F}}{\pi^{2}}\left(\frac{11}{3} C_{A}-\frac{4}{3} T n_{l}\right) \ln \frac{M_{t}^{2}}{\mu_{\mathrm{hard}}^{2}}+C_{F}\left(\frac{1}{3} C_{F}+\frac{1}{2} C_{A}\right) \ln \frac{M_{t}^{2}}{\mu_{\mathrm{fac}}^{2}}\right] .
$$

The consistency of the "direct matching" procedure ensures that $C_{1}$ does not contain any energydependent terms. We would like to point out that the factorization scale dependence of $\operatorname{Im}\left[\mathcal{A}_{1}\right]$ is cancelled by the factorization scale dependence in $C_{1}$ up to a small term $\propto C_{F} \alpha_{s} \frac{\Gamma_{t}}{M_{t}} \ln \left(\frac{M_{t}}{\mu_{\mathrm{fac}}}\right)$ (see also [22]). This term remains as a consequence of our ignorance of a consistent treatment of the finite

\footnotetext{
${ }^{4}$ For $\alpha_{s} \ll v \ll 1$, i.e. far away from the threshold regime, no distinction between soft and hard scales is needed.
} 

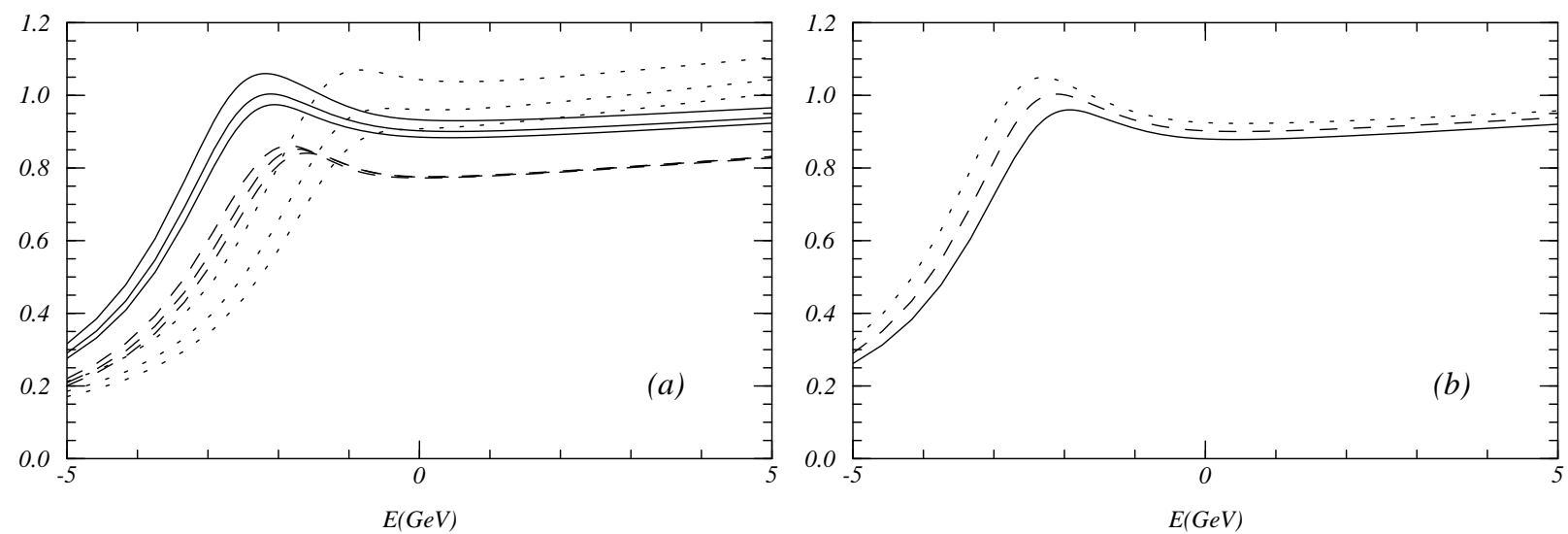

Figure 1: (a) The total normalized photon-mediated $t \bar{t}$ cross section at LO (dotted lines), NLO (dashed lines) and NNLO (solid lines) for the soft scales $\mu_{\text {soft }}=50$ (upper lines), 75 and $100 \mathrm{GeV}$ (lower lines). (b) The NNLO cross section for $\alpha_{s}\left(M_{z}\right)=0.115$ (solid line), 0.118 (dashed line) and 0.121 (dotted line). More details and the other parameters are given in the text.

width effects at the NNLO level. Due to the small size of this contribution, however, the corresponding ambiguity can be ignored for the examination of the relativistic NNLO corrections determined in this paper. The LO cross section can be recovered from the NNLO one in Eq. (5) by taking into account only the dominant contributions in the third line of Eq. (17) and setting $C_{1}=1, C_{2}=0$, whereas the NLO cross section can be obtained by incorporating also the $\mathcal{O}\left(\alpha_{s}\right)$ corrections to the Coulomb potential and to the constant $C_{1}$. In Fig. 1(a) the LO (dotted lines), NLO (dashed lines) and NNLO (solid lines) normalized cross sections are plotted versus $E$ in the range $-5 \mathrm{GeV}<E<5 \mathrm{GeV}$ for $M_{t}=175 \mathrm{GeV}, \alpha_{s}\left(M_{z}\right)=0.118$ and $\Gamma_{t}=1.43 \mathrm{GeV}$. For the scales the choices $\mu_{\text {soft }}=50$ (upper lines), 75 and $100 \mathrm{GeV}$ (lower lines) and $\mu_{\text {hard }}=\mu_{\text {fac }}=M_{t}$ have been made and two-loop running of the strong coupling has been used. It is evident that the NNLO corrections are large. Compared to the NLO cross section, the $1 S$ peak is shifted towards smaller energies by several hundred MeV and the large negative NLO corrections for positive energies are compensated to some extent. Whereas the location of the $1 S$ peak is quite insensitive to changes in the soft scale, the residual dependence of the normalization of the NNLO cross section on the soft scale $\mu_{\text {soft }}$ is not improved at all compared to the NLO cross section. In fact, for energies above the $1 S$ peak it is worse for the NNLO cross section than for the NLO one. The dependence of the NNLO cross section on the hard scale $\mu_{\text {hard }}$ and the factorization scale $\mu_{\mathrm{fac}}$ are, on the other hand, much smaller and, therefore, not displayed here. The behavior of the NNLO corrections clearly indicates that the convergence of the perturbative series for the $t \bar{t}$ cross section is much worse than expected from the general arguments given by Fadin and Khoze [1]. For the normalization of the cross section we estimate, at least at the present stage, a theoretical uncertainty at the level of five to ten percent. For comparison, in Fig. 1(b) the NNLO cross section is displayed for $\alpha_{s}\left(M_{z}\right)=0.115$ (solid line), 0.118 (dashed line) and 0.121 (dotted line) and $\mu_{\text {soft }}=75 \mathrm{GeV}$. The other parameters are chosen as before. A more detailed examination of the NNLO contributions will be carried out in a future publication.

This work is supported in part by the U.S. Department of Energy under contract No. DOE DEFG03-90ER40546. 


\section{References}

[1] V. S. Fadin and V. A. Khoze, Pis'ma Zh. Eksp. Teor. Fiz. 46 (1987) 417 [JETP Lett. 46 (1987) 525]; Yad. Fiz. 48 (1988) 487 [Sov. J. Nucl. Phys. 48 (1988) 309].

[2] M. J. Strassler and M. E. Peskin, Phys. Rev. D 43 (1991) 1500.

[3] W. Kwong, Phys. Rev. D 43 (1991) 1488.

[4] M. Jeżabek, J. H. Kühn and T. Teubner, Z. Phys. C 56 (1992) 653.

[5] Y. Sumino, K. Fujii, K. Hagiwara, H. Murayama and C.-K. Ng, Phys. Rev. D 47 (1993) 56.

[6] K. Fujii, T. Matsui and Y. Sumino, Phys. Rev. D 50 (1994) 4341.

[7] E. Accomando et al., the ECFA/DESY LC Physics Working Group, Physics with $e^{+} e^{-}$linear colliders, DESY 97-100, hep-ph/9705442.

[8] M. S. Berger, proceedings of the "Workshop on Physics at the First Muon Collider and the Front End of a Muon Collider", Fermilab, November 6-9, 1997, hep-ph/9712486.

[9] M. Jezabek, J. H. Kühn, Phys. Lett. B 316 (1993) 360 and references therein;

R. Harlander, M. Jezabek and J. H. Kühn, Act. Phys. Pol. B 27 (1996) 1781.

[10] W. Fischler, Nucl. Phys. B 129 (1977) 157.

[11] A. Billoire, Phys. Lett. B 92 (1980) 343.

[12] W. E. Caswell and G. E. Lepage, Phys. Lett. B 167 (1986) 437.

[13] A. H. Hoang, proceedings of the "Workshop on Physics at the First Muon Collider and the Front End of a Muon Collider", Fermilab, November 6-9, 1997, hep-ph/9801273.

[14] A. H. Hoang, Phys. Rev. D 57 (1998) 1615.

[15] M. Jeżabek and T. Teubner, Z. Phys. C 59 (1993) 669.

[16] W. Mödritsch and W. Kummer, Nucl. Phys. B 430 (1994) 3; Phys. Lett. B 349 (1995) 525.

[17] P. Labelle, McGill Report No. McGill-96/33, hep-ph/9608491.

[18] B. Grinstein and I. Z. Rothstein, Phys. Rev. D 57 (1998) 78.

[19] M. Peter, Phys. Rev. Lett. 78 (1997) 602; Nucl. Phys. B 501 (1997) 471.

[20] S. N. Gupta and S. F. Radford, Phys. Rev. D 24 (1981) 2309, ibid. 25 (1982) 3430 (Erratum);

S. N. Gupta, S. F. Radford and W. W. Repko, Phys. Rev. D 26 (1982) 3305.

[21] E. H. Wichmann and C. H. Woo, J. Math. Phys. 2 (1961) 178;

L. Hostler, J. Math. Phys. 5 (1964) 591;

J. Schwinger, J. Math. Phys. 5 (1964) 1606.

[22] A. H. Hoang, Phys. Rev. D 56 (1997) 5851. 
[23] G. T. Bodwin, E. Braaten and G. P. Lepage, Phys. Rev. D 51 (1995) 1125.

[24] G. Källen and A. Sabry, K. Dan. Vidensk. Selsk. Mat.-Fys. Medd. 29 (1955) No. 17, 1.

[25] A. H. Hoang, Phys. Rev. D 56 (1997) 7276.

[26] A. Czarnecki and K. Melnikov, University of Karlsruhe Report No. TTP97-54, hep-ph/9712222.

[27] A. H. Hoang, J. H. Kühn and T. Teubner, Nucl. Phys. B 452 (1995) 173.

[28] M. B. Voloshin, Int. J. Mod. Phys. A 10 (1995) 2865.

[29] S. G. Karshenboim, Yad. Fiz. 56 (1993) 155 [Sov. J. Nucl. Phys. 56 (1993) 1710]. 